\title{
Interval Type II Fuzzy Sliding Mode Controller Tuned With PSO Designed for Attitude Control of Micro Aircraft Vehicle
}

\author{
Xiang-jian Chen ${ }^{1}$, $\mathrm{Di} \mathrm{Li}^{2}$ and Zhihong $\mathrm{Lu}^{2}$ \\ ${ }^{1}$ Jiangsu University of Science and Technology, School of Computer Science and \\ Engineering, China \\ ${ }^{2}$ China Shipbuilding Industry Corporation 723, China \\ cxj831209@163.com,lee_di@163.com
}

\begin{abstract}
MAV (Micro Aerial Vehicle) is nonlinear plant, it is difficult to obtain stable control for MAV attitude due to uncertainties. The purpose of this paper is to propose one robust 、 stable control strategy for MAV to accommodate system uncertainties, variations, and external disturbances. In this paper, an interval type II fuzzy sliding-mode controller combined with PSO algorithm (ITIIFSMC-PSO) is proposed for MAV attitude control system. The proposed ITIIFSMC-PSO is a combination of the interval type II fuzzy logic control (ITIIFLC) and the sliding-mode control (SMC) which inherits the benefits of these two methods, furthermore, PSO algorithm was adopted to tune the type II fuzzy controller's antecedent and consequent parameters for the plant control. The objective of the controller is to ensure the asymptotic stability of the closed-loop system. The Lyapunov stability method is adopted to verify the stability of the ITIIFSMC-PSO system. At last, a Quadrotor MAV with uncertainty and disturbance are adopted to illustrate the validity of the proposed method. The simulation results show that the ITIIFSMC-PSO achieves the best tracking performance in comparison with the interval type II Fuzzy logic controller (ITIIFLC), the interval type II fuzzy sliding-mode controller (ITIIFSMC).
\end{abstract}

Keywords: Interval type II fuzzy sliding mode controller, PSO, Lyapunov stability theory, MAV

\section{Introduction}

One of the most important problems in Micro Aircraft Vehicle design is that of attitude stabilization and control [1-3]. Although the missions of space vehicles and their attitude requirements vary greatly, high pointing accuracy is an important part of the overall design problem for a Micro Aircraft Vehicle control system. Meeting the Micro Aircraft Vehicle attitude control system design requirements in a realistic environment where the knowledge about the system parameters may be incomplete, disturbances are present and orbital operations induces structural vibrations in the flexible appendages, is a challenging task for the designers.

Significant research efforts have been made for the problem of stabilization and performance of Micro Aircraft Vehicle attitude control system. Intelligent control theory represented by fuzzy control [4], neural network control [5], and their integration with modern robust control theory represented by adaptive control [6], sliding mode control [7] provides effective ways to solve the modeling and control of Micro Aircraft Vehicle with characteristics of time-variant, serious nonlinearity, parameter and structure uncertainty, and external disturbances in most cases which make it very difficult to realize high accuracy control for the attitude angle by conventional methods. For resolving this problem. The FSMC [8-11], a hybrid of the SMC and FLC, gives a simple 
way to design the controller systematically and provides the asymptotical stability of the system. In general, the FSMC can also reduce the rule number in the FLC and still possess robustness in the face of model uncertainties and external disturbances.

Type II fuzzy logic systems (T2-FIS) are used successfully in many application areas, and these include control, classification, etc. [12,13], and there is an increasing interest in the research and implementations of T2-FIS because they offer more advantages in handling uncertainty with respect to type-1 fuzzy systems [14]. Type II fuzzy sets, characterized by membership grades that are themselves fuzzy, were introduced by Zadeh in 1975 [15] to better handle uncertainties. Type II FLSs [16] can better handle system uncertainties and have the potential to outperform their type- 1 counterparts. TO date, type II FLSs have been used successfully in many applications, for example, data and survey processing [17], word modeling [18-19], time-series forecasting [20,17], decision making [21], communication and networks [22,23], plant monitoring and diagnostics [24], etc. Even though fuzzy control is the most widely used application of fuzzy set theory, a literature search reveals that only a few type II FLSs are employed in the field of control. Interval type II FLCs were applied to mobile robot control [18], quality control of sound speakers [26], connection admission control in ATM networks [27]. T2FNNs has also been proposed and used in plant control [28-30]

The proposed ITIIFSMC-PSO is a combination of ITIIFLC and SMC, and also inherits the benefits of these two methods which can reduce the number of rules dramatically, furthermore, PSO algorithm[31,32] was adopted to tune the type II fuzzy controller's antecedent and consequent parameters for the plant control. The proposed ITIIFSMCPSO controller can also provide more robustness than the conventional FLC to handle the uncertainty and disturbance.

This paper is organized as follows. The attitude dynamic model of MAV is given in section2. In Section 3, the SMC of a nonlinear system is reviewed. The design procedure of the ITIIFLC is addressed in detail in Section 4. In section 5, The PSO algorithm was introduced to tune the type II fuzzy controller's antecedent and consequent parameters for the plant control. In Section 6, the proposed ITIIFSMC-PSO is described and the Lyapunov function is utilized to testify the stability of the proposed method. In Section 7, a Quadrotor MAV with uncertainty and disturbance are adopted to examine the feasibility and effectiveness of the proposed IT2FSMC-PSO. The conclusions are drawn in Section 8.

\section{Attitude Dynamic Model of Micro Aircraft Vehicle}

Attitude angular velocity dynamic equation under torque $U$ for Micro Aircraft Vehicle has been achieved in the body fixed frame according to the reference [1-3], which is given as following:

$$
J \dot{w}=-\Omega J w+U+d
$$

Where $J \in R^{3 \times 3}$ denotes the rotational inertia matrix of Micro Aircraft Vehicle;

$w=[p, q, r]^{T}$ denotes the angular velocity of Micro Aircraft Vehicle; $U=\left[U_{1}, U_{2}, U_{3}\right]^{T}$ denotes the control torque in the body fixed frame; $d=\left[d_{1}, d_{2}, d_{3}\right]^{T}$ denotes the external disturbance torque, matrixes $J, \Omega$ are defined respectively as:

$$
J=\left[\begin{array}{ccc}
I_{x} & 0 & 0 \\
0 & I_{y} & 0 \\
0 & 0 & I_{z}
\end{array}\right], \Omega=\left[\begin{array}{ccc}
0 & -r & -q \\
r & 0 & -p \\
-q & p & 0
\end{array}\right]
$$

Organizing above equations, we obtain: 


$$
\left\{\begin{array}{l}
\dot{p}=\frac{U_{1}-q r\left(I_{z}-I_{y}\right)}{I_{x}} \\
\dot{q}=\frac{U_{2}-p r\left(I_{x}-I_{z}\right)}{I_{x}} \\
\dot{r}=\frac{U_{3}-p q\left(I_{y}-I_{x}\right)}{I_{z}}
\end{array}\right.
$$

It is not difficult to obtain the relationship between attitude angular velocity and three angular velocity components in the body fixed frame through the transformation matrix between body fixed frame and earth fixed frame, the attitude angular dynamic equation is given as:

$$
\left[\begin{array}{c}
\dot{\phi} \\
\dot{\theta} \\
\dot{\psi}
\end{array}\right]=\left[\begin{array}{ccc}
1 & \sin \phi \tan \theta & \cos \phi \tan \theta \\
0 & \cos \phi & -\sin \phi \\
0 & \sin \phi / \cos \theta & \cos \phi / \cos \theta
\end{array}\right]\left[\begin{array}{l}
p \\
q \\
r
\end{array}\right]
$$

where $\phi, \theta, \psi$ represent the roll, pitch and yaw angels in the inertial reference frame.

Making the outputs track the attitude of Micro Aircraft Vehicle by designing the control torque U. Arranging the above dynamic equations for designing the control scheme conveniently, the attitude control model of Micro Aircraft Vehicle obtained can be expressed as:

$$
\left\{\begin{array}{l}
\dot{x}_{1}=b T \\
\dot{T}=F(T)+U+d \\
y=x_{1}
\end{array}\right.
$$

Where $\varphi=\left[\begin{array}{lll}\theta & \psi & \phi\end{array}\right]^{T}, w=\left[\begin{array}{lll}q & r & p\end{array}\right]^{T}, F(T)=\left[\begin{array}{lll}\frac{\left(I_{x}-I_{z}\right) p r}{I_{y}} & \frac{\left(I_{x}-I_{y}\right) p q}{I_{z}} & \frac{\left(I_{z}-I_{y}\right) q r}{I_{x}}\end{array}\right]^{T}$,

$$
U=\left[\begin{array}{lll}
U_{2} & U_{3} & U_{1}
\end{array}\right]^{T}, b\left(x_{1}\right)=\left[\begin{array}{ccc}
\cos \phi & -\sin \phi & 0 \\
\sin \phi / \cos \theta & \cos \phi / \cos \theta & 0 \\
\sin \phi \tan \theta & \cos \phi \tan \theta q & 1
\end{array}\right] .
$$

Next, propose $x=b T, f=\dot{b} F(T)+b T$, the control model turned to as following eventually:

$$
\left\{\begin{array}{l}
\ddot{x}=f+\tilde{f}+(b+\tilde{b}) U+d \\
y=x
\end{array}\right.
$$

where $x_{1}, x_{2}, x \in R^{3}$ denotes controlled state vector, $F, f \in R^{3}$ means system function vector, $b \in R^{3 \times 3}$ means control coefficient matrix, $U \in R^{3}$ means control input matrix vector, $d \in R^{3}$ means the disturbance vector, $y \in R^{3}$ means the control output vector, $\tilde{f}, \tilde{b} \in R^{3 \times 3}$ means uncertainty function vector caused by attitude perturbations, each component expression of $f=\left[\begin{array}{lll}f_{1} & f_{2} & f_{3}\end{array}\right]^{T}$ referred to as: 


$$
\left\{\begin{aligned}
f_{1}= & (p+q \tan \theta \sin \varphi+r \tan \theta \cos \varphi)(-r \cos \varphi-q \sin \varphi) \\
& -\frac{I_{x}-I_{y}}{I_{z}} p q \sin \varphi+\frac{I_{z}-I_{x}}{I_{y}} r p \cos \varphi \\
f_{2}= & \frac{1}{\cos \theta}((p+q \tan \theta \sin \varphi+r \tan \theta \cos \varphi)(q \cos \varphi-r \sin \varphi) \\
& +\tan \theta(q \sin \varphi+r \cos \varphi)(q \sin \varphi-r \cos \varphi) \\
& \left.+\frac{I_{x}-I_{y}}{I_{z}} p q \sin \varphi+\frac{I_{z}-I_{x}}{I_{y}} r p \cos \varphi\right) \\
f_{3}= & \frac{1}{\cos ^{2} \theta}(q \sin \varphi+r \cos \varphi)(q \sin \varphi-r \cos \varphi) \\
& +\tan \theta(p+q \tan \theta \sin \varphi+r \tan \theta \cos \varphi)(q \cos \varphi-r \sin \varphi) \\
& +\frac{I_{y}-I_{z}}{I_{x}} r q+\frac{I_{x}-I_{y}}{I_{z}} p q \cos \varphi \tan \theta+\frac{I_{z}-I_{x}}{I_{y}} r p \sin \varphi \tan \theta
\end{aligned}\right.
$$

Aerodynamic characteristics parameters will get perturbations resulting from flying conditions or flying attitude changed. At the same time, external gust of wind and flow could not be ignored. Thus, control system of Micro Aircraft Vehicle is one MIMO nonlinear system with uncertainty and perturbations.

\section{Sliding Model Control}

Consider a nonlinear system:

$$
\frac{d^{n} x}{d t^{n}}=f(x, E)+g(x, F) u+d(t)
$$

where $x(t)=\left(x, \dot{x}, \cdots, x^{(n)}\right)^{T}$ is the state vector, $\mathrm{u}$ is the control input of the plant, $\mathrm{d}(\mathrm{t})$ is the matched disturbance with known bound, $\|d(t)\| \leq D, E \in[\underline{E}, \bar{E}]$ and $F \in[\underline{F}, \bar{F}]$ are interval matrices with proper dimensions and bounded by $\|\underline{E}\| \leq\|E\| \leq\|\bar{E}\|$ and $\|\underline{F}\| \leq\|F\| \leq\|\bar{F}\|$, respectively.

The tracking problem of (8) is to find a suitable control law to let the states follow a desired trajectory $x_{d}(t)$. The tracking error is defined as

$$
e(t)=x(t)-x_{d}(t)=\left(e, \dot{e}, \cdots, e^{(n-1)}\right)^{T}
$$

then, the sliding surface $s(e, t)=0$ is defined as

$$
s(e, t)=\sum_{k=0}^{n-1}\left(\begin{array}{c}
n-1 \\
k
\end{array}\right) \lambda^{k} e^{(n-k-1)}
$$

where $\lambda$ is a strictly positive real constant. A suitable control $u$ has to be found so as to retain the error $e(t)$ on the sliding surface $s(e, t)=0$. To achieve this purpose, a positive Lyapunov function $\mathrm{V}$ is defined as

$$
V=\frac{1}{2} s^{2}
$$


One sufficient condition for the stability of the system is given by

$$
\dot{V}=\frac{1}{2} \frac{d}{d t} s^{2} \leq-\eta|s|
$$

which leads to the convergence condition:

$$
s \dot{s} \leq-\eta|s| \rightarrow \dot{s} \operatorname{sgn}(s) \leq-\eta
$$

If $\eta>0,(12)$ states that the system is driven to the sliding surface. If the state trajectory has reached the sliding surface $\mathrm{s}=0$, then it simultaneously slides into the origin $\mathrm{e}=0$ despite the matched parametric uncertainties and disturbances.

Then, the first step is to select the parameter $\lambda$ properly. The next step is to find the control law that will keep the system in sliding mode. Eq. (13) gives a sufficient condition for the asymptotic stability of the closed-loop system. we take the time derivative of $s(e, t)$ as:

$$
\begin{aligned}
\dot{s}(t) & =\frac{d}{d t}(s(e, t))=\frac{d}{d t}\left[\sum_{k=0}^{n-1}\left(\begin{array}{c}
n-1 \\
k
\end{array}\right) \lambda^{k} e^{(n-k-1)}\right] \\
& =f(x, E)+g(x, F) u+d(t)-x_{d}^{(n)}+\sum_{k=0}^{n-1}\left(\begin{array}{c}
n-1 \\
k
\end{array}\right) \lambda^{k} e^{(n-k)}
\end{aligned}
$$

Combining (14) with (13), we can get:

$$
\left\{f(x, E)+g(x, F) u+d(t)-x_{d}^{(n)}+\sum_{k=0}^{n-1}\left(\begin{array}{c}
n-1 \\
k
\end{array}\right) \lambda^{k} e^{(n-k)}\right\} \operatorname{sgn}(s) \leq-\eta
$$

The sliding control law $\mathrm{u}$ is now chosen as:

$$
\begin{array}{r}
u=\hat{g}^{-1}[G(\hat{u}-k(x, t) \operatorname{sgn}(s))-\hat{f}\rfloor \\
\hat{u}=x_{d}^{(n)}-\sum_{k=0}^{n-1}\left(\begin{array}{c}
n-1 \\
k
\end{array}\right) \lambda^{k} e^{(n-k)}
\end{array}
$$

Where $\hat{f}, \hat{g}$ are estimating functions of $\mathrm{f}$ and $\mathrm{g}$, respectively. The bounds are defined as the following:

$$
0<\beta_{\min } \leq g \hat{g}^{-1} \leq \beta_{\max }
$$

Choose the gain $\mathrm{G}$ as

$$
G=\left(\beta_{\min } \beta_{\max }\right)^{\left(-\frac{1}{2}\right)}
$$

It remains to find $k(x, t)$ so as to satisfy the convergence condition (13). Now we substitute (17) and (11) for (15) to obtain 


$$
\begin{aligned}
& \left\{f(x, E)+g \hat{g}^{-1}[G(\hat{u}-k(x, t) \operatorname{sgn}(s))-\hat{f}]+d(t)-x_{d}^{(n)}+\sum_{k=0}^{n-1}\left(\begin{array}{c}
n-1 \\
k
\end{array}\right) \lambda e^{k} e^{(n-k)}\right\} \operatorname{sgn}(s) \leq-\eta \\
& \rightarrow\left[\left(f-g \hat{g}^{-1} \hat{f}\right)+g \hat{g}^{-1} G \hat{u}-\hat{u}-g \hat{g}^{-1} k(x, t) \operatorname{sgn}(s)+d\right] \operatorname{sgn}(s) \leq \eta \\
& \rightarrow\left[\Delta f+\left(g \hat{g}^{-1} G-1\right) \hat{u}+d\right] \operatorname{sgn}(s)-g \hat{g}^{-1} G k(x, t) \leq \eta
\end{aligned}
$$

a sufficient condition for the control law to fulfill the sliding surface $\mathrm{s}=0$ is

$$
k(x, t) \geq \beta(D+R+\eta)+(\beta-1) U
$$

where $\beta=\left(\beta_{\max } / \beta_{\min }\right)^{(1 / 2)}$

\section{Interval Type II Fuzzy Logic System}

In this section, a brief overview of type II fuzzy systems is presented.A type II fuzzy set $\tilde{A}$

$$
\widetilde{A}=\left\{\left((x, u), \mu_{\tilde{A}}(x, u)\right) \mid \forall x \in X, \forall u \in J x \subseteq[0,1]\right\}
$$

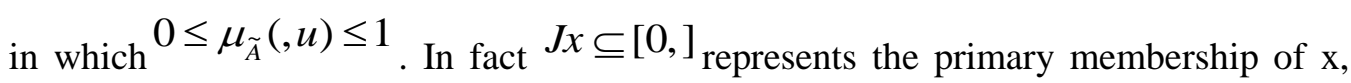
and $\mu_{\tilde{A}}(x, u)$ is a type-1 fuzzy set known as the secondary set. Hence, a type II membership grade can be any subset in $[0,1]$, the primary membership, and corresponding to each primary membership, there is a secondary membership that defines the possibilities for the primary membership. Uncertainty is represented by a region, which is called the footprint of uncertainty (FOU). When we have an interval type II membership function. The uniform shading for the FOU represents the entire interval type II fuzzy set and it can be described in terms of an upper membership function $\bar{\mu}_{\tilde{A}}(x)$ and a lower membership function $\underline{\mu}_{\tilde{A}}(x)$.

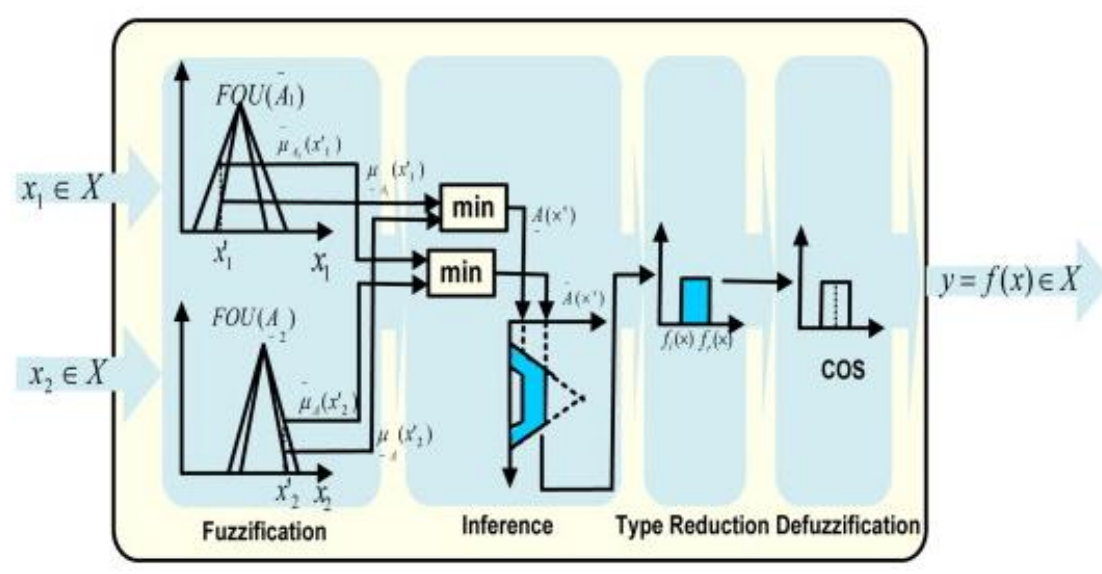

Figure 1. Structure of Interval Type II Fuzzy Logic System

A type II FLS is characterized by IF-THEN rules, where their antecedent or consequent sets are now of type II. type II FLSs, can be used when the circumstances are too uncertain to determine exact membership grades such as when the training data is affected by noise. Similarly, to the type-1 FLS, a type II FLS includes a fuzzifier, a rule base, 
fuzzy inference engine, and an output processor, as we can see in Figure 1. Now we explain each of the blocks shown in Figure 1.

The fuzzifier maps a numeric vector $x=\left(x_{1}, \cdots, x_{p}\right)^{T}$ into a type II fuzzy set $\tilde{A}_{x}$ in $\mathrm{X}$ [13], an interval type II fuzzy set in this case. $\tilde{A}_{x}$ is a type II fuzzy singleton if $\mu_{\tilde{A}_{x}}(x)=1 / 1$ for $x=x^{\prime}$ and $\mu_{\tilde{A}_{x}}(x)=1 / 0$ for all other $x \neq x^{\prime}$. The structure of rules in a type-1 FLS and a type II FLS is the same, but in the latter the antecedents and the consequents is represented by type II fuzzy sets. So for a type II FLS with $\mathrm{p}$ inputs $x=\left(x_{1}, \cdots, x_{p}\right)^{T}$ and one output $y \in Y$, Multiple Input Single Output (MISO), if we assume there are M rules, the lth rule in the type II FLS can be written down as follows:

$$
\begin{aligned}
& R^{1}: I F \quad x_{1} \text { is } \tilde{F}_{1}^{l} \quad \text { and } \cdots \text { and } x_{p} i s \tilde{F}_{p}^{l} \text { THEN } y \text { is } \\
& \tilde{G}^{l} l=1, \cdots, M
\end{aligned}
$$

In the type II FLS, the inference engine combines rules and gives a mapping from input type II fuzzy sets to output type II fuzzy sets. It is necessary to compute the join $\sqcup$ (unions) and the meet $\Pi$ (intersections), as well as the extended sup-star compositions (sup star compositions) of type II relations. If $\tilde{F}_{1}^{l} \times \cdots \tilde{F}_{p}^{l}=\tilde{A}^{l}$ then (22) can be re-written as follows:

$$
R^{l}: \tilde{F}_{1}^{l} \times \cdots \tilde{F}_{p}^{l} \rightarrow \tilde{G}^{l}=\tilde{A}^{l} \rightarrow \tilde{G}^{l} \quad l=1, \cdots, M
$$

$R^{l}$ is described by the membership function $\mu_{R^{l}}(x, y)=\mu_{R^{l}}\left(x_{1}, \cdots, x_{p}, y\right)$

where $\mu_{R^{l}}(x, y)=\mu_{\widetilde{A}^{l} \rightarrow \widetilde{G}^{l}}(x, y)$

In the FLS, we used interval type II fuzzy sets and intersection under product t-norm, so the result of the input and antecedent operations, which is an interval type-1 set,

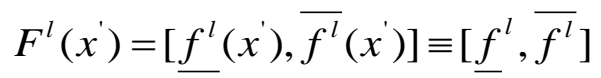

where

$$
\underline{f^{l}}\left(x^{\prime}\right)=\underline{\mu}_{\widetilde{F}_{p}^{l}}\left(x_{1}^{\prime}\right) * \cdots * \underline{\mu}_{\widetilde{F}_{p}^{l}}\left(x_{p}^{\prime}\right)
$$

And

$$
\overline{f^{l}}\left(x^{\prime}\right)=\bar{\mu}_{\tilde{F}_{p}^{l}}\left(x_{1}^{\prime}\right) * \cdots * \bar{\mu}_{\tilde{F}_{p}^{l}}\left(x_{p}^{\prime}\right)
$$

where $*$ stands for the product operation. Next, the type-reducer generates a type-1 fuzzy set output. This type-1 fuzzy set is also an interval set, for the case of our FLS we used center of sets (cos) type reduction, which is expressed as

$$
\begin{gathered}
Y_{\cos }(x)=\left[y_{l}, y_{r}=\int_{y^{1} \in\left[y_{l}^{1}, y_{r}^{1}\right]} \cdots \int_{y^{M} \in\left[y_{l}^{M}, y_{r}^{M}\right]} \int_{f^{1} \in\left[\underline{f}^{1}, \bar{f}^{1}\right]} \cdots\right. \\
\left.\times \int_{f^{M} \in\left[\underline{f}^{M}, \bar{f}^{M}\right]} \frac{1}{\sum_{i=1}^{M} f^{i} y^{i} / \sum_{i=1}^{M} f^{i}}\right]
\end{gathered}
$$


This interval set is determined by its two end points, $y_{l}, y_{r}$, which corresponds to the centroid of the type II interval consequent set $\widetilde{G}^{i}$

$$
\begin{gathered}
C_{\widetilde{G}^{i}}=\int_{\theta_{1} \in J_{y 1}} \cdots \int_{\theta_{N} \in J_{y N}} 1 /\left(\sum_{i=1}^{N} y_{i} \theta_{i} / \sum_{i=1}^{N} \theta_{i}\right)=\left[y_{l}^{i}, y_{r}^{i}\right] \\
y_{l}=\frac{\sum_{i=1}^{M} f_{l}^{i} y_{l}^{i}}{\sum_{i=1}^{M} f_{l}^{i}}, y_{r}=\frac{\sum_{i=1}^{M} f_{r}^{i} y_{r}^{i}}{\sum_{i=1}^{M} f_{r}^{i}}
\end{gathered}
$$

The values of $y_{l}, y_{r}$ define the output interval of the type II fuzzy system, which can be used to verify if training or testing data are contained in the output of the fuzzy system. From the type-reducer (21), we obtain an interval set $Y_{\text {cos }}$, to defuzzify it we use the average of $y_{l}, y_{r}$, so the defuzzified output of an interval singleton type II FLS is

$$
u_{\text {ITIIFLC }}=y(x)=\left(y_{l}+y_{r}\right) / 2
$$

\section{Particle Swarm Optimization}

In this section, PSO algorithm is applied to FLC design for a two axis robot arm. The objective in here is to tune all parameters (the fuzzy controller's antecedent and consequent parameters) of the Mamdani type FLC through a given algorithm ${ }^{[24]}$ to control the given trajectory.

\subsection{PSO Algorithm}

The PSO concept consists of changing the velocity (or accelerating) of each particle toward its pbest and the gbest position at each time step. Each particle tries to modify its current position and velocity according to the distance between its current position and pbest, and the distance between its current position and gbest as shown the following. At each step $\mathrm{n}$, by using the individual best position, pbest, and global best position, gbest, a new velocity for the ith particle is updated by

$$
V_{i}(n)=\chi\left(V_{i}(n-1)+\varphi_{1} r_{1}\left(\text { pbest }_{i}-P_{i}(n-1)\right)+\varphi_{2} r_{2}\left(\text { gbest }_{i}-P_{i}(n-1)\right)\right.
$$

where each particle represents a potential solution and has a position represented by a position vector $p_{i}, r_{1}$ and $r_{2}$ are random numbers between 0 and $1 ; \varphi_{1}, \varphi_{2}$ are positive constant learning rates; $\chi$ is called the constriction factor and is defined by (32).

$$
\chi=\frac{2}{\left|2-\varphi-\sqrt{\varphi^{2}-4 \varphi}\right|}, \varphi=\varphi_{1}+\varphi_{2}, \varphi>4
$$

The velocity is confined within the range of $\left[-v_{\max }, v_{\max }\right]$. If the velocity violates these limits, it is forced to its proper values. Changing velocity by this way enables the ith particle to search around its local best position, pbest, and global best position, gbest. Based on the updated velocity, each particle changes its position as following:

$$
p_{i}(n)=p_{i}(n-1)+V_{i}(n)
$$




\subsection{Optimization Of FLC With PSO}

If there are $\mathrm{M}^{*} \mathrm{~N}$ parameters to be optimized in this study. PSO searches all of the antecedent and consequent parameters in $\mathrm{w}$ dimensional spaces.

The order of a particle is shown as the following

$$
p_{i}=\left\lfloor\sigma_{11} c_{11} \cdots \sigma_{1(N * M)} c_{1(N * M)} \cdots \sigma_{(N * M) 1} c_{(N * M) 1} \cdots \sigma_{(N * M)(N * M)} c_{(N * M)(N * M)}\right\rfloor
$$

where the parameters $\sigma_{i j}, c_{i j}$ represent the center and deviation of the Gaussian MFs. In the above equation, the first line and the second line constitute the parameters of the first controller and the second controller, respectively. The initial values of particles are randomly generated in the first generation.

The most crucial step in applying PSO is to choose the best cost functions which are used to evaluate fitness of each particle. In during tuning process with PSO, one cost functions are used as Mean of Root of Squared Error (MRSE). The cost functions for ith particle are computed as follows:

$$
\operatorname{MRSE}=E(k)=\frac{1}{N} \sum_{i=1}^{N}|e(i)|+|u(i)|
$$

Where e(i) is the trajectory error of ith sample for the object, $\mathrm{N}$ is the number of sample, $\mathrm{k}$ is the iteration number, $\mathrm{u}(\mathrm{i})$ is the control signal, respectively. All parameters of the FLC are updated at every final time $\left(t_{f}\right)$.

\section{Interval Type II Fuzzy Sliding Mode Controller with PSO}

\subsection{Design of PSO Based Interval Type II Fuzzy Sliding Mode Controller}

Based on the ITIIFLC mentioned in the previous subsection, the design procedures for IT2FSM-PSO will be described in this subsection. The structure of an IT2FSM-PSO system is shown in Figure 2.

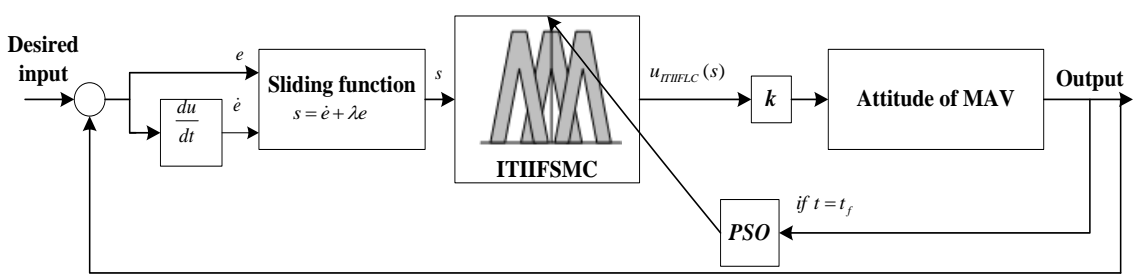

Figure 2. The Architecture of ITIIFSMC-PSO Based Control System

Referring to (16), (17) and (30), the control law for IT2FSMC is now chosen as

$$
u=\hat{g}^{-1} G \hat{u}-\hat{g}^{-1} \hat{f}-\hat{g}^{-1} G u_{\text {ITIFLC }}(s)
$$

\subsection{Stability of ITIIFSMC-PSO}

In order to analyze the closed-loop system stability, we adopt the same Lyapunov function as in (4). With the convergence condition (6), we obtain

$$
\left\{f+g\left[\hat{g}^{-1} G \hat{u}-\hat{g}^{-1} \hat{f}-\hat{g}^{-1} G u_{\text {ITIFLC }}(s)\right]+d(t)-x_{d}^{(n)}+\sum_{k=0}^{n-1}\left(\begin{array}{c}
n-1 \\
k
\end{array}\right) \lambda^{k} e^{(n-k)}\right\} \operatorname{sgn}(s) \leq-\eta
$$

which implies that 


$$
\left\lfloor\Delta f+\left(1-\beta^{-1}\right) \hat{u}-\beta^{-1} u_{\text {ITIIFL }}(s)+d\right\rfloor \operatorname{sgn}(s) \leq-\eta
$$

To achieve asymptotic stability, we consider

When $\mathrm{s}>0$

$$
\beta^{-1} u_{\text {ITIFL }}(s) \geq(\Delta f+d+\eta)+\left(1-\beta^{-1}\right) \hat{u}
$$

which implies that

$$
\begin{array}{cc}
\left.u_{\text {ITIIFL }}\right|_{\max } \geq \beta(R+D+\eta)+(\beta-1) U & \text { When } \mathrm{s}<0 \\
\beta^{-1} u_{\text {ITIIFL }}(s) \leq-(\Delta f+d+\eta)-\left(1-\beta^{-1}\right) \hat{u} &
\end{array}
$$

which implies that

$$
\left.u_{\text {ITIFL }}\right|_{\max } \leq-[\beta(R+D+\eta)+(\beta-1) U]
$$

We have $u_{\text {ITIFL }}(s)$ being positive (negative) if $\mathrm{s}$ is positive (negative). Thus, Eq. (37) implies $\dot{V} \leq 0$ which completes the stability verification of the IT2FSMC-PSO.

We can summarize the design procedure for ITIIFSMC-PSO as follows:

Step 1.Determine a stable sliding surface s from (8), (9) and (10).

Step 2.Drive the equivalent sliding control u from (15).

Step 3.Design ITIIFSMC-PSO with sliding function s.

Step 4.Regulation the fuzzy rule from (34).

Step 5.Imply the fuzzy implication from (25)、 (26) and (27).

Step 6.Calculate the overall control u from (36).

Step 7.Adjust the parameters if necessary.

\section{Experimental Results}

The Quadrotor Micro Aircraft Vehicle is characterized by a nominal main body inertia matrix:

$$
J=\left[\begin{array}{ccc}
0.954 & 0 & 0 \\
0 & 0.545 & 0 \\
0 & 0 & 0.926
\end{array}\right] k g-m^{2}
$$

The initial attitude angle set to be zero $\theta_{0}=0, \phi_{0}=0, \psi_{0}=0$. The attitude perturbations set to be sine function for reflecting the changed attitude perturbations real-time, the perturbations given as: $\tilde{\theta}=0.3^{\circ} \sin \frac{\pi t}{4}, \tilde{\psi}=0.5^{\circ} \sin \frac{\pi t}{8}, \tilde{\phi}=0.2^{\circ} \sin \frac{\pi t}{3}$; Considering the torque perturbations caused by Micro Aircraft Vehicle body and Micro Aircraft Vehicle wing, the uncertainty functions set to be $\left[0.2^{\circ} \cos t, 0.15^{\circ} \cos t, 0.1^{\circ} \cos t\right]^{T}$; The external flow has been taken into account in the model used for simulating at $\left[0.3^{\circ} \sin t, 0.2^{\circ} \sin t, 0.1^{\circ} \sin t\right]^{T}$. The control objective is to control the attitude angle to tracking the reference trajectory shown as:

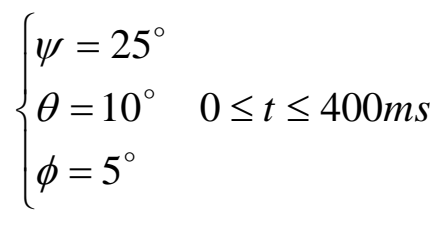


In this example, we apply Interval Type II Fuzzy Logic controller (ITIIFLC), Interval Type II Fuzzy Sliding Mode controller (ITIIFSMC) and Interval Type II Fuzzy Sliding Mode controller based on PSO (ITIIFSMC-PSO) to the Quadrotor MAV plant. Figure 3 shows the trajectory tracking error response curve of three angles under the circumstance without external disturbance and uncertainty. Figure 4 shows the trajectory tracking error response curve of three angles under the circumstance with external disturbance and uncertainty. Subscript Numbers under attitude Angles corresponding to three kinds of controller: " 1 " is a representative of ITIIFLC controller; " 2 " is a representative of ITIIFSMC controller; " 3 " is a representative of ITIIFSMC-PSO controller.

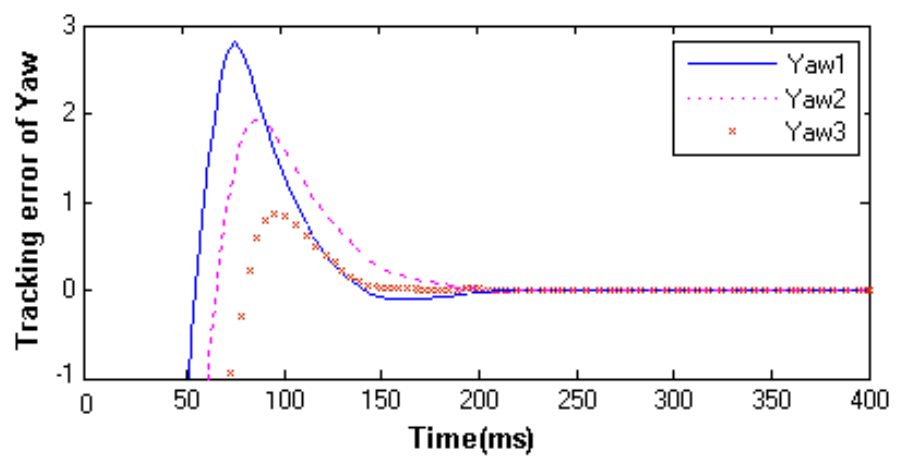

Figure 3-A. Tracking Error Curves of Pitch Angle $\psi$

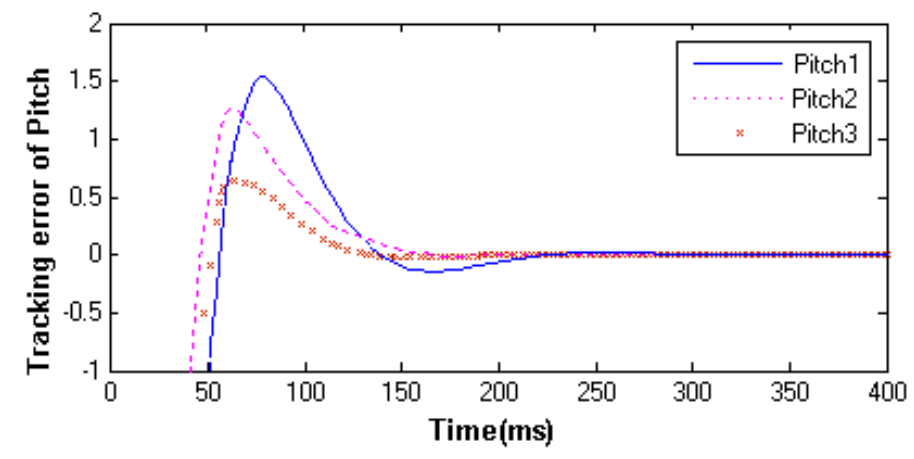

Figure 3-B. Tracking Error Curves of Yaw Angle $\theta$

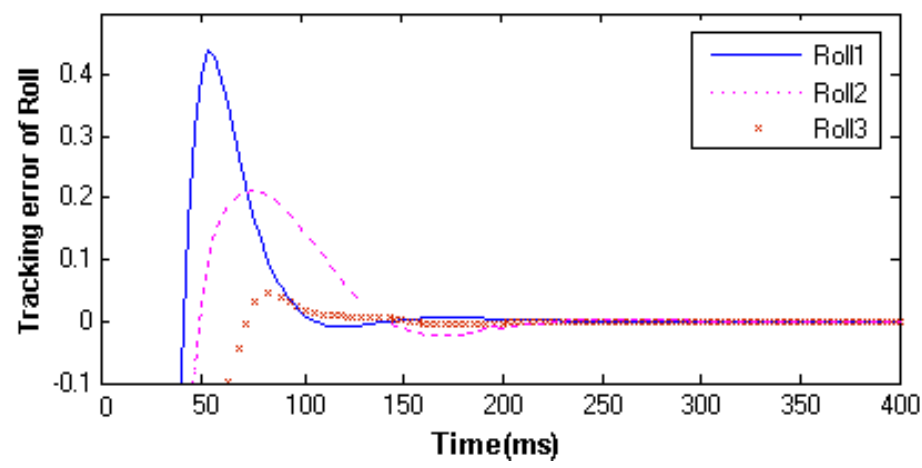

Figure 3-C. Tracking Error Curves of Roll Angle $\phi$

Figure 3. Tracking Error Curves of Attitude Angles 


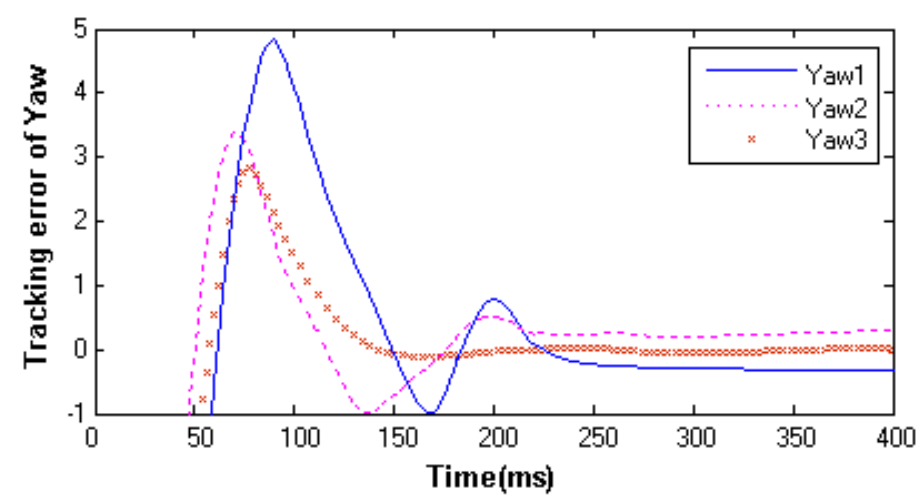

Figure 4-A Tracking Error Curves Of Pitch Angle $\psi$

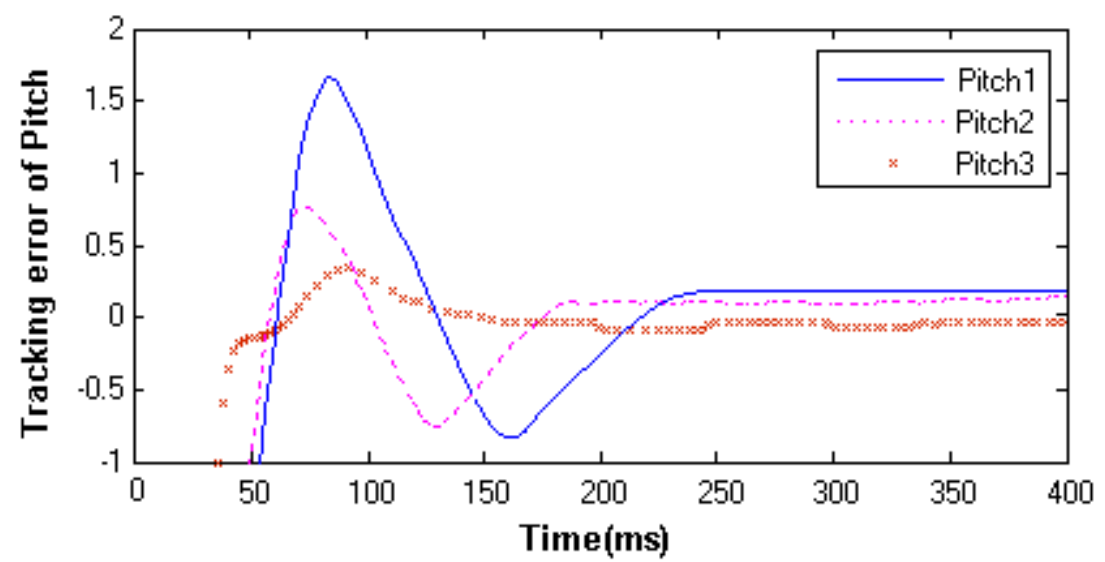

Figure 4-B. Tracking Error Curves Of Yaw Angle $\theta$

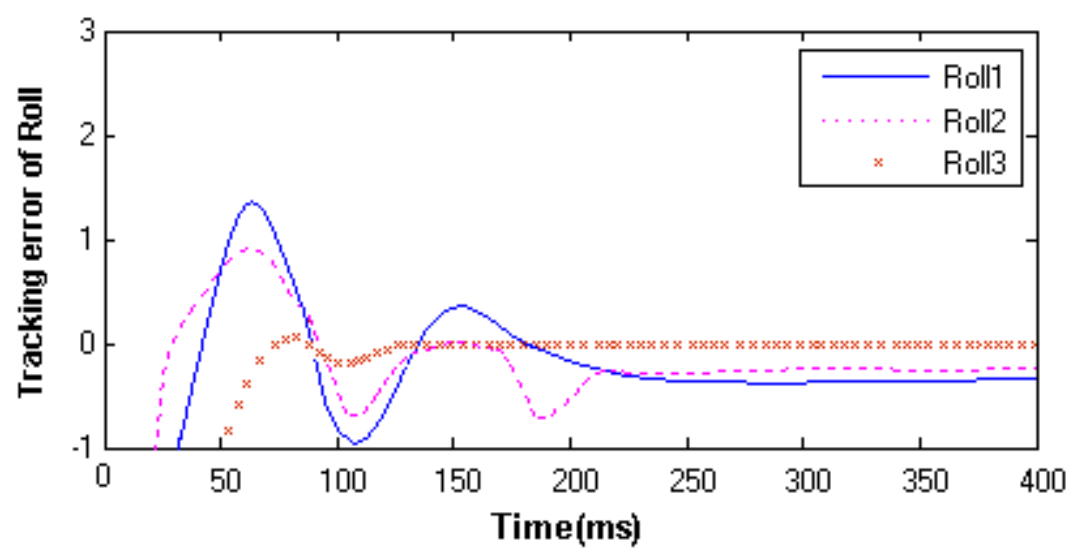

Figure 4-C. Tracking Error Curves Of Roll Angle $\phi$

Figure 4. Tracking Error Curves of Attitude Angles

The above two experimental results indicate that tracking performances can be guaranteed under three different control scheme. Conventional adaptive sliding mode control scheme which responses fast, but its overshoot is somewhat of big; Interval type II fuzzy logic controller which has smaller steady-state error, but with longer regulation time; Interval type II fuzzy sliding mode controller which has better control accuracy and smaller overshoot. The control accuracy decreased under uncertainty factors and external 
disturbances for all of the three control schemes, but which could satisfy the performance standard of control system, furthermore, the Interval type II fuzzy sliding mode controller based on PSO gives better performances compared with the other ones.

Arranging the above simulation results from Figure 3-Figure 4, then the response data of three attitude angles are listed in Table 1.

Table 1. Response Data of Three Attitude Angles

\begin{tabular}{|c|c|c|c|}
\hline & Regulation time(ms) & $\begin{array}{c}\text { Overshoot }(\% \\
)\end{array}$ & Steady-state error $\left(^{\circ}\right)$ \\
\hline Yaw1 & $180 / 245$ & $14 / 24.5$ & $0.15 / 0.25$ \\
\hline Yaw2 & $175 / 220$ & $9.5 / 17$ & $0.08 / 0.30$ \\
\hline Yaw3 & $145 / 150$ & $4.5 / 14$ & $0.02 / 0.03$ \\
\hline Pitch1 & $205 / 240$ & $15.5 / 18.5$ & $0.075 / 0.15$ \\
\hline Pitch2 & $150 / 175$ & $12.5 / 17.5$ & $0.045 / 0.25$ \\
\hline Pitch3 & $120 / 145$ & $1.5 / 4.0$ & $0.030 / 0.05$ \\
\hline Roll1 & $190 / 250$ & $8.8 / 28$ & $0.04 / 0.25$ \\
\hline Roll2 & $185 / 210$ & $4.4 / 18$ & $0.01 / 0.40$ \\
\hline Roll3 & $100 / 120$ & $1 / 3$ & $0.01 / 0.02$ \\
\hline
\end{tabular}

From Figure 3-Figure 4 and Table 1, we can see that not only from nominal system, but also from system with uncertainty and external disturbances in Figure 4, it can be seen that Interval Type II Fuzzy Logic controllerproduces bigger overshoot and steadystate error; Interval Type II Fuzzy Sliding Mode controller produces big overshoot and small steady-state error; Interval Type II Fuzzy Sliding Mode controller based on PSO gets smaller overshoot and small steady-state error.

\section{Conclusion}

This paper introduced the PSO based tuning method for ITIIFSMC controller to control the attitude of MAV. The all parameters concerning the fuzzy controller were determined using PSO algorithm. In order to examine effects of the cost functions on the controller parameter optimalization. The Lyapunov stability theorem has been used to testify to the asymptotic stability of the closed-loop system. For attitude of MAV control system, the proposed ITIIFSMC-PSO can make the pole track a desired sinusoidal angular displacement in the presence of parameter variations and disturbances. From the simulation results, the ITIIFSMC-PSO can give the best tracking performance compared with ITIIFLC and ITIISMC.

\section{References}

[1] P. Castillo, A. Dzul and R. Lozano, "Real-time Stabilization and Tracking of a Four-Rotor Mini Rotorcraft", IEEE Trans. Control Syst. 2004, 12(4):510-517.

[2] P. Castillo, R. Lozano and A. Dzul, "Stabilization of a mini rotorcraft having four rotors", IEEE Contr. Syst. Mag. 2005, 25: 45-55.

[3] K. Jinhyun, S.K. Min, P. Sangdeok, "Accurate modeling and robust hovering control for a Qaud-rotor VTOL aircraft”, J Intell Robot Syst. 2010, 57:9-26.

[4] S.L. Zhou, P. Han, D.F. Wang, "Fuzzy-neural net based control strategy for robot manipulator trajectory tracking, in: International Conference on Machine Learning and Cybernetics", 2003, 1:596-601.

[5] A. Das, Lewis and K. Subbarao, "Backstepping approach for controlling a quadrotor using Lagrange form dynamics", J Intell Robot Syst. 2009; 56:127-151.

[6] E. Johnson, S. Kannan, "Adaptive trajectory control for autonomous helicopters", AIAA J. Guid. Control Dyn. 2005, 28:524-528.

[7] M.B. Cheng, V. Radisavljevic and W.C. Su, "Slding model boundary control of a parabolic PDE system with parameter variations and boundary uncertainties", Automatica, 2011, 47(2):381-387.

[8] H. Alli and O. Yakut, "Fuzzy sliding-mode control of structures,Engineering Structures", Volume 27, Issue 2, January 2005, Pages 277-284. 
[9] H.F. Ho, Y.K. Wong and A.B. Rad, "Adaptive fuzzy sliding mode control with chattering elimination for nonlinear SISO systems.Simulation Modelling Practice and Theory”, Volume 17, Issue 7, August 2009, Pages 1199-1210.

[10] S. Wang, L. Hou, L. Dong and H. Xiao, "Adaptive Fuzzy Sliding Mode Control of Uncertain Nonlinear SISO Systems", Procedia Engineering, Volume 24, 2011, Pages 33-37.

[11] N. Yagiz and Y. Hacioglu, "Robust control of a spatial robot using fuzzy sliding modes", Mathematical and Computer Modelling, Volume 49, Issues 1-2, January 2009, Pages 114-127

[12] M. Biglarbegian, W.W. Melek and J.M.Mendel, "Design of novel interval type II fuzzy controllers for modular and reconfigurable robots: theory and experiments", IEEE Transactions on Industrial Electronics Volume 58, Issue 4,2011, Pages 1371-1384.

[13] J.R. Castro, O. Castillo, P. Melin, L.G. Martinez, S. Escobar and I. Camacho, "Building fuzzy inference system with the interval type II fuzzy logic toolbox", Advances in Soft Computing Volume 41,2007, Pages 53-62.

[14] J.M. Mendel, "Uncertainty Rule Based Fuzzy Logic Systems: Introduction and New Directions", Prentice-Hall, Upper-Addle River, 2001.

[15] L.A. Zadeh, "The concept of a linguistic variable and its application to approximate reasoning-1", Information Sciences, Volume 8, 1975, Pages 199-249

[16] O. Castillo, "Patricia Melin. A review on the design and optimization of interval type II fuzzy controllers", Applied Soft Computing, Volume 12, Issue 4, April 2012, Pages 1267-1278

[17] J.M. Mendel, "Rule-Based Fuzzy Logic Systems: Introduction and New Directions', Prentice-Hall, Englewood Cliffs, NJ, 2001.

[18] J.M. Mendel, "Fuzzy sets for words: A new beginning", Proceedings of the IEEE International Conference on Fuzzy Systems Volume 1, 2003, Pages 37-42

[19] H. Wu and J.M.Mendel, "Antecedent connector word models for interval type II fuzzy logic systems", Proceedings of the IEEE International Conference on Fuzzy Systems Volume 2, 2004, Pages 1099-1104

[20] Q. Liang and J. M. Mendel, "Interval type II fuzzy logic systems: theory and design", IEEE Trans. Fuzzy Syst. Volume 8, Issue 5, 2000, Pages 535-550.

[21] T. Ozen, J. Garibaldi and S. Musikasuwan, "Modelling the variation in human decision making", Proceedings of the IEEE International Conference on Fuzzy Systems Volume 2, 2004, Pages 617-622.

[22] Q. Liang, and J.M. Mendel, "Equalization of nonlinear time-varying channels using type II fuzzy adaptive filters", IEEE Trans. Fuzzy Syst. Volume 8, 2000, Pages 551-563.

[23] Q. Liang and J.M. Mendel, "MPEG VBR video traffic modelling and classification using fuzzy technique”, IEEE Trans. Fuzzy Syst.Volume 9, 2001, Pages 183-193.

[24] O. Castillo and P. Melin, "A new hybrid approach for plant monitoring and diagnostics using type II fuzzy logic and fractal theory", Proceedings of the IEEE International Conference on Fuzzy Systems Volume 1, 2003, Pages 102-107.

[25] H.A. Hagras, "A hierarchical type II fuzzy logic control architecture for autonomous mobile robots", IEEE Trans. Fuzzy Syst. Volume 12, 2004, Pages 524-539.

[26] P. Melin and O. Castillo, "Intelligent control of nonlinear dynamic plants using type II fuzzy logic and neural networks. Proceedings of NAFIPS, 2002, Pages 22-27.

[27] Q. Liang, N. Karnik and J.M. Mendel, "Connection admission control in ATM networks using survey based type II fuzzy logic systems", IEEE Trans. Syst Man Cybern. Volume 30, Issue 3, 2000, Pages 329-339.

[28] F.J. Lin, S.Y. Chen, P.H. Chou, P.H. Shieh, "Interval type II fuzzy neural network control for X-YTheta motion control stage using linear ultrasonic motors", Neurocomputing, Volume 72, Issues 4-6, January 2009, Pages 1138-1151

[29] C.S. Chen, W.C. Lin, "Self-adaptive interval type II neural fuzzy network control for PMLSM drives", Expert Systems with Applications, Volume 38, Issue 12, November-December 2011, Pages 1467914689

[30] O. Mendoza, P. Melín and O. Castillo, "Interval type II fuzzy logic and modular neural networks for face recognition applications", Applied Soft Computing, Volume 9, Issue 4, September 2009, Pages 1377-1387

[31] Z. Bingül, O. Karahan, "A Fuzzy Logic Controller tuned with PSO for 2 DOF robot trajectory control”, Expert Systems with Applications, Volume 38, Issue 1, January 2011, Pages 1017-1031

[32] P. Melin, F. Olivas, O. Castillo, F. Valdez, J. Soria and M. Valdez, "Optimal design of fuzzy classification systems using PSO with dynamic parameter adaptation through fuzzy logic.", Expert Systems with Applications, Volume 40, Issue 8, 15 June 2013, Pages 3196-3206

[33] M.Y. Hsiao, T.H.S. Li, J.Z. Lee, C..H.Chao and S.H. Tsai, "Design of interval type-2 fuzzy slidingmode controller. Information Sciences”, Volume.178, 2008, Pages 1696-1716 